\title{
Potential Neutrino Signals from Galactic $\gamma$-Ray Sources
}

\author{
Alexander Kappes \\ Friedrich-Alexander-University Erlangen-Nuremberg, Erlangen, Germany \\ kappes@physik.uni-erlangen.de \\ Jim Hinton \\ School of Physics and Astronomy, The University of Leeds, Leeds, UK \\ Max-Planck-Institut für Kernphysik, Heidelberg, Germany \\ Landessternwarte, Universität Heidelberg, Königstuhl, Heidelberg, Germany \\ Christian Stegmann \\ Friedrich-Alexander-University Erlangen-Nuremberg, Erlangen, Germany \\ Felix A. Aharonian \\ Dublin Institute for Advanced Studies, Dublin, Ireland \\ Max-Planck-Institut für Kernphysik, Heidelberg, Germany
}

\begin{abstract}
The recent progress made in Galactic $\gamma$-ray astronomy using the High Energy Stereoscopic System (H.E.S.S.) instrument provides for the first time a population of Galactic $\mathrm{TeV} \gamma$-ray, and hence potential neutrino sources, for which the neutrino flux can be estimated. Using the energy spectra and source morphologies measured by H.E.S.S., together with new parameterisations of pion production and decay in hadronic interactions, we estimate the signal and background rates expected for these sources in a first-generation water Cherenkov detector (ANTARES) and a next generation neutrino telescope in the Mediterranean Sea, KM3NeT, with an instrumented volume of $1 \mathrm{~km}^{3}$. We find that the brightest $\gamma$-ray sources produce neutrino rates above $1 \mathrm{TeV}$, comparable to the background from atmospheric neutrinos. The expected event rates of the brightest sources in the ANTARES detector make a detection unlikely. However, for a $1 \mathrm{~km}^{3}$ KM3NeT detector, event rates of a few neutrinos per year from these sources are expected, and the detection of individual sources seems possible. Although generally these estimates should be taken as flux upper limits, we discuss the conditions and type of $\gamma$-ray sources for which the neutrino flux predictions can be considered robust.
\end{abstract}


Subject headings: neutrinos, neutrino telescopes, pulsar wind nebulae, supernova remnants, binary systems, very high energy gamma-rays

\section{Introduction}

Neutrinos and $\gamma$-rays in the $\mathrm{GeV}-\mathrm{PeV}$ range provide the only available probes of cosmic ray $(\mathrm{CR})$ acceleration sites and $\mathrm{CR}$ propagation in our Galaxy. Both particles are produced in hadronic interactions of cosmic protons and nuclei with the ambient gas and are not deflected in the interstellar magnetic field. Despite many years of research, no source of high-energy cosmic neutrinos has yet been identified (AMANDA Coll. 2005; BAIKAL Coll. 1999). This experimental situation is unfortunate, as the detection of even a single cosmic TeV neutrino could potentially provide compelling evidence for the long sought-after class of hadronic $\mathrm{CR}$ accelerators. On the other hand, $\mathrm{TeV} \gamma$-ray astronomy has recently made considerable progress with the detection of several shell-type supernova remnants (SNRs), the prime candidates for the acceleration of Galactic CRs (see, e.g., Hillas (2005) for a recent review). As $\mathrm{TeV}$ neutrinos should be produced in roughly equal numbers to $\mathrm{TeV}$ photons in any hadron accelerator, these objects represent prime targets for neutrino telescopes. Moreover, the recent High Energy Stereoscopic System (H.E.S.S.) (Hinton 2004) survey of the Galactic plane (H.E.S.S. Coll. 2005b, 2006a) has revealed several additional classes of potential high energy Galactic neutrino sources: X-ray binaries, pulsar wind nebulae (PWNe), and unidentified but probable hadron-accelerating sources such as HESS J1303-631 (H.E.S.S. Coll. 2005g), as well as diffuse emission from the interactions of CRs in the Galactic center (GC) region (H.E.S.S. Coll. 2006b).

The case for CR acceleration in SNRs was strengthened by the detection of resolved TeV $\gamma$-ray emission from the shells of two SNRs (H.E.S.S. Coll. 2004b, 2005e). However, while these measurements provide direct evidence of particle acceleration in these objects, in both cases an ambiguity remains between electrons and hadrons as the radiating particles. Both inverse Compton scattering of relativistic electrons and decay of neutral pions produced in hadronic interactions, can satisfactorily explain the available data. In fact, the only instance in which a very strong case for very high-energy (VHE) $\pi^{0}$-decay emission can be made is for the giant molecular clouds of the GC region, due to the strong spatial correlation between $\gamma$-ray emission and available target material (interstellar gas). However, even in this case the nature of the CR source remains uncertain, as at least two good candidates exist: the SNR Sgr A East (Crocker et al. 2005b) and the black hole Sgr A* (Aharonian \& Neronov 2005). The case for hadron acceleration in unidentified $\gamma$-ray sources can be made based on a lack of associated X-ray emission, which would be expected for cosmic electron accelerators. 
Some of the brightest sources in the TeV $\gamma$-ray sky are PWNe, for example, the nebulae associated with the powerful Crab and Vela pulsars (HEGRA Coll. 2004; H.E.S.S. Coll. 2006c). The $\gamma$-ray emission from these objects is normally interpreted as inverse Compton upscattering of cosmic microwave background radiation (or synchrotron) photons by energetic electrons. However, the existence of a significant fraction of nuclei in pulsar winds has been suggested (Hoshino et al. 1992). In some scenarios, the TeV emission may be dominated by the decay of pions produced in the interactions of these nuclei, and significant production of neutrinos may occur (see, e.g., Horns et al. (2006); Bednarek (2003); Amato et al. $(2003))$.

Finally, $\mathrm{TeV} \gamma$-ray emission has recently been reported from the X-ray binaries LS 5039 (H.E.S.S. Coll. 2005c) and LS I +61 303 (MAGIC Coll. 2006). The rapid cooling of TeV electrons in the dense radiation fields associated with such objects is suggestive of a hadronic origin for the $\gamma$-radiation. While the $\gamma$-ray emission from these sources is relatively weak, they are promising candidates for neutrino emission because of the strong $\gamma$-ray absorption that likely occurs within the sources (Aharonian et al. 2006).

Given these recent advances in our knowledge of the $\mathrm{TeV} \gamma$-ray emission of Galactic sources, and the planned or current construction of major TeV neutrino detectors, we consider this an appropriate time to consider in detail the detectability of neutrino counterparts to these sources in the medium term. Since H.E.S.S. measures $\gamma$-ray spectra up to $10 \mathrm{TeV}$ and in some cases even above $10 \mathrm{TeV}$, the prediction for $\mathrm{TeV}$ neutrino signals can be done quite robustly, especially for extended sources for which the internal $\gamma$-ray absorption is negligible.

In contrast to the search for extra-Galactic neutrino sources, the geographic location of the detector is of vital importance to a search for Galactic hadron accelerators. In the northern hemisphere, Cassiopeia A is the only known $\gamma$-ray SNR and has a rather weak TeV flux $\left(\sim 2 \times 10^{-12} \mathrm{erg} \mathrm{cm}^{-2} \mathrm{~s}^{-1}, 1-10 \mathrm{TeV}\right)$ (HEGRA Coll. 2001). The unidentified northern hemisphere source TeV J2032+4130 is also rather weak (HEGRA Coll. 2005), and for the extended TeV source reported in Saz Parkinson et al. (2005), the spectrum is unknown so far. On the other hand, the strongest northern hemisphere $\gamma$-ray source, the Crab Nebula, with a measured energy spectrum exceeding $50 \mathrm{TeV}$, is very likely an electron accelerator (HEGRA Coll. 2004). This leaves the recently detected $\gamma$-ray emitter, the X-ray binary LS I +61 303, as the only possible strong Galactic neutrino source so far, providing that the $\gamma$-ray emission is caused by pion decay and that strong $\gamma$-ray absorption occurs.

The situation in the southern hemisphere is strikingly different: at least five SNRs have now been detected, and two, RX J1713.7-3946 and RX J0852.0-4622, are extremely bright $\left(\sim 10^{-10} \mathrm{erg} \mathrm{cm}^{-2} \mathrm{~s}^{-1}, 0.5-10 \mathrm{TeV}\right)$ (H.E.S.S. Coll. 2004b, 2005e). In addition, the southern hemisphere contains the unique collection of objects at the GC and many as yet unidentified 
sources. The probability of detection of a Galactic neutrino source by a (downward-looking) Northern Hemisphere detector therefore appears much greater than for a Southern Hemisphere instrument. In this paper we discuss in detail the observability of neutrino emissions from CR accelerators in the ANTARES detector (ANTARES Coll. 1999), currently under construction in the Mediterranean Sea, and an additional planned $\mathrm{km}^{3}$ scale detector in the Mediterranean Sea, KM3NeT (Katz 2006) (in this paper we assume for KM3NeT an instrumented volume of $1 \mathrm{~km}^{3}$ ). Figure 1 shows the sky map of known $\mathrm{TeV} \gamma$-ray sources and their visibility to the major Northern and Southern Hemisphere neutrino telescopes. Since it is not possible with current neutrino telescopes to separate neutrinos and anti-neutrinos the word neutrino is used to encompass both throughout the article.

\section{Galactic $\gamma$-ray emission}

\subsection{Resolved sources}

The H.E.S.S. survey of the inner Galaxy (H.E.S.S. Coll. 2006a), taken together with targeted observations of southern hemisphere Galactic sources, provides a comprehensive list of VHE $\gamma$-ray sources relevant to the current work. Earlier claims of $\gamma$-ray emission from southern Galactic sources have either been confirmed (e.g., H.E.S.S. Coll. (2004b)) or refuted (e.g., H.E.S.S. Coll. (2005f)). Table 1 lists all 27 southern hemisphere Galactic sources reported so far by the H.E.S.S. collaboration. Based on information in the H.E.S.S. publications and other relevant multi-wavelength information, we assign each source into one of four categories: (A) Unambiguously associated with a SNR shell, (B) associated with a binary system, (C) lacking any good counterpart at other wavelengths and (D) plausibly associated with a PWN. While the assignment of some objects is rather arbitrary (particularly between categories $\mathrm{C}$ and $\mathrm{D}$ ), and some assignments will undoubtedly change as better multi-wavelength data appear, we nevertheless consider that the objects of category A, and perhaps also category $\mathrm{C}$, are most likely candidates for neutrino emission. The most promising objects are, of course, young shell-type SNRs. The morphological and spectrometric characteristics of $\mathrm{TeV} \gamma$-ray emission from two objects of this category, RX J1713.7-3946 and RX J0852.0-4622, have been studied by H.E.S.S. with great detail. At $1 \mathrm{TeV}$, both sources show fluxes as large as the Crab Nebula flux but with significantly harder energy spectra extending beyond $10 \mathrm{TeV}$. If the hadronic $\gamma$-rays from interactions of $\mathrm{CR}$ with the ambient matter dominate over the inverse Compton component, then the neutrino fluxes from the shells of these objects can be calculated with a good accuracy in the most relevant energy band between 0.1 and $100 \mathrm{TeV}$.

For some objects with angular sizes less than $\sim 0.2^{\circ}$, an affiliation with a particular 
category is ambiguous as a discrimination between shell-like emission and that of any central nebula is difficult. HESS J1813-178 is one such object, with a shell-like radio emission (Brogan et al. 2005), a central X-ray nebula (Ubertini et al. 2005), and an unresolved TeV $\gamma$-ray emission (H.E.S.S. Coll. 2006a).

Category B and D objects are generally treated as leptonic (inverse Compton) sources and therefore are less likely neutrino emitters. However, interpretations of $\mathrm{TeV} \gamma$-ray emission from two representatives of these classes, namely, the microquasar LS 5039 and the plerion Vela X, in terms of hadronic interactions are quite possible (see, e.g., Aharonian et al. (2006); Horns et al. (2006)). In this paper, neutrino fluxes are calculated for all objects in classes $\mathrm{A}, \mathrm{B}, \mathrm{C}$ and D.

\subsection{Undetected hard-spectrum sources}

Experiments such as H.E.S.S. have substantially reduced energy flux sensitivities beyond $\sim 10 \mathrm{TeV}$ relative to their $1 \mathrm{TeV}$ performance. For this reason bright sources with extremely hard spectra between $\sim 100 \mathrm{TeV}$ and $1 \mathrm{PeV}$ could in principle be "missed" by current Cherenkov telescopes. Due to the rapid rise of the effective collection area of neutrino telescopes with energy, such sources could be promising candidates for these detectors. It is instructive to consider how hard the energy spectrum of a source must be to be undetected in $\mathrm{TeV} \gamma$-rays and potentially detectable in $\sim 100 \mathrm{TeV}$ neutrinos. The H.E.S.S. survey of the Galactic plane reaches a typical sensitivity of $1 \times 10^{-13} \mathrm{~cm}^{-2} \mathrm{~s}^{-1}$ above $10 \mathrm{TeV}$. To produce a detectable flux in a $\mathrm{km}^{3}$ volume neutrino detector and be missed by this survey, we estimate that a photon index of at least about 1 is required. In addition to the Cherenkov telescope limits, air-shower arrays have produced limits on ultra-high-energy $\gamma$-ray fluxes for most of the sky. The CASA-MIA detector was used to produce limits on northern hemisphere point sources of $\sim 4 \times 10^{-14} \mathrm{~cm}^{-2} \mathrm{~s}^{-1}$ above $140 \mathrm{TeV}$ (McKay et al. 1993). The SPASE detector at the South Pole produced limits over the region $\delta<-45^{\circ}$ of $\sim 2 \times 10^{-13} \mathrm{~cm}^{-2} \mathrm{~s}^{-1}$ above $50 \mathrm{TeV}$ (van Stekelenborg et al. 1993). Updated results from the larger area and longer inte-

gration time of the SPASE-2 instrument (Dickinson et al. 2000) may be useful in constraining the $(>50 \mathrm{TeV})$ extrapolation of the spectra of strong southern hemisphere sources such as RX J0852.0-4622 in the future. 


\subsection{Diffuse emission and unresolved sources}

For instruments with modest angular resolution and a very wide field of view, it may be easier to detect large-scale diffuse emission than to resolve individual sources. This fact is demonstrated by the recent MILAGRO detection of a diffuse $\mathrm{TeV} \gamma$-ray signal from the Galactic plane between $l=40^{\circ}$ and $100^{\circ}$ (MILAGRO Coll. 2005). Any such signal must be considered as the sum of the emission induced by CR (hadron and electron) interactions with a contribution from unresolved sources. The calculation of diffuse neutrino and $\gamma$-ray emission arising from CR interactions in our Galaxy is rather complex (see, e.g., Strong et al. (2004)). However, at least for the emission from CR hadrons, a reasonable approximation simplifies the situation enormously: for constant emissivity, the $\gamma$-ray and neutrino signals are simply proportional to the product of the total column depth of material (molecular +

atomic) with the mean CR density in the volume integrated. Following Aharonian (2001) the emissivity for CRs is such that

$$
\begin{aligned}
F_{\gamma}\left(>E_{\gamma}\right) & =4 \times 10^{-14} S\left(\frac{E_{\gamma}}{1 \mathrm{TeV}}\right)^{-1.7} \mathrm{~cm}^{-2} \mathrm{~s}^{-1} \\
\text { with } S & =\left(\frac{\Omega}{1 \mathrm{deg}^{2}}\right)\left(\frac{N_{H}}{10^{22} \mathrm{~cm}^{-2}}\right)
\end{aligned}
$$

assuming that the locally measured CR spectrum is valid throughout the Galaxy. While this assumption may be valid in regions far from active CR accelerators, enhanced emission is expected in the neighbourhood of such sources. The relatively high $\gamma$-ray flux detected from the giant molecular clouds of the GC region (H.E.S.S. Coll. 2006b) implies an enhanced CR density in the central $200 \mathrm{pc}$ of our Galaxy. However, this GC emission is still rather weak $\left(10^{-11} \mathrm{erg} \mathrm{s}^{-1}\right.$ in the $0.2-20 \mathrm{TeV}$ range), making a detection of the associated neutrino emission rather difficult. The integrated diffuse emission from the entire Inner Galaxy (diffuse plane emission) may present a more promising target. In the window $|b|<1^{\circ},|l|<30^{\circ}$, the approximate mean molecular and atomic column densities are $4 \times 10^{22}$ and $1.5 \times 10^{22} \mathrm{~cm}^{-2}$, respectively (Dame et al. 2001). Assuming an enhancement factor of 1.4 of the CR density in the inner Galaxy relative to the local density (Hunter at al. 1997), the predicted diffuse flux is $\sim 3.5 \times 10^{-11} \mathrm{~cm}^{-2} \mathrm{~s}^{-1}(>1 \mathrm{TeV})$, with a spectral index close to that of the parent spectrum, i.e., $\sim 2.7$. As this calculation assumes no contribution from electrons above $1 \mathrm{TeV}$ (as suggested by more detailed models from (Strong et al. 2004)), this flux is valid for both $\gamma$-rays and neutrinos.

An estimate of the contribution from unresolved sources requires a population model. While the number of detected sources is relatively small, a meaningful comparison with simple population models is possible. We consider here a model (discussed also in H.E.S.S. Coll. $(2006 \mathrm{a}))$ in which mono-luminous sources are distributed within the Galaxy roughly as the 
distribution of molecular material. This simple approach is sufficient to explain the latitudinal and flux distributions of the detected sources. This simple model suggests that the unresolved component represents $\sim 30 \%-50 \%$ of the total flux. Equally uncertain is the fraction of the detected sources in which neutral pion decay is the dominant $\gamma$-ray production mechanism. The fraction of the total detected $\gamma$-ray flux represented by source classes A, B and $\mathrm{C}$ in the region $|b|<1^{\circ},|l|<1^{\circ}$ is $\sim 50 \%$ (a sub-area of this region with $|b|<0.3^{\circ}$ and $|l|<0.8^{\circ}$ is later referred to as the "Galactic center ridge"). Diffuse $\gamma$-ray and neutrino fluxes above $1 \mathrm{TeV}$ from this region are therefore $\sim 3 \times 10^{-11}$ and $\sim 1.5 \times 10^{-11} \mathrm{~cm}^{-2} \mathrm{~s}^{-1}$, respectively. The spectral index of the unresolved class is most likely close to that of the detected sources, i.e., $\sim 2.3$. The harder spectral index of the source component makes any neutrino detection of diffuse emission likely to be dominated by unresolved sources.

Applying the same method to the region $40^{\circ}<l<100^{\circ},|b|<5^{\circ}$ results in a flux of $4 \times 10^{-10} \mathrm{~cm}^{-2} \mathrm{~s}^{-1}$, compatible with the MILAGRO measurement in this region: $F(>1 \mathrm{TeV})=(5.1 \pm 1.0 \pm 1.7) \times 10^{-10} \mathrm{~cm}^{-2} \mathrm{~s}^{-1} \mathrm{sr}^{-1}$ (MILAGRO Coll. 2005). The expected contribution from unresolved sources is $\sim 60 \%$ in this case. As the region is not very visible for Mediterranean detectors it is not discussed further in this paper.

\section{Neutrino fluxes}

In $\gamma$-ray sources where the $\mathrm{TeV}$ emission is dominated by the decay of $\pi^{0}$ particles produced in $p$ - $p$ interactions, the measured $\gamma$-ray spectra can be used to derive the expected $\mathrm{TeV}$ neutrino spectra. Several calculations of expected neutrino spectra have been made for the special case of a power-law spectrum of primary hadrons (Berezinskv \& Volvnskv 1979; Gaisser 1990; Crocker et al. 2005a; Alvarez-Muñiz \& Halzen 2002; Costantini \& Vissani 2005; Lipari 2006). However, given that for many sources curved spectra are measured (or expected) in the energy range of neutrino detectors $(E \approx 1 \mathrm{TeV}$ to $E>1 \mathrm{PeV}$ ), a revised treatment of such spectra is required.

A recent parameterisation of the pion and secondary particle production in hadronic interactions (based on results from the SIBYLL event generator; Fletcher et al. (1994)) can be used to derive the $\gamma, \nu_{e}$, and $\nu_{\mu}$ spectra for arbitrary incident proton spectra (Kelner et al. 2006). We have used these parameterisations to calculate the relationship between $\gamma$-ray and neutrino spectra in the case in which the primary proton spectrum is given by a power-law with index $\alpha$ and an exponential cut-off energy $\epsilon_{p}$ :

$$
\frac{\mathrm{N}_{p}}{\mathrm{E}_{p}}=k_{p}\left(\frac{E_{p}}{1 \mathrm{TeV}}\right)^{-\alpha} \exp \left(-\frac{E_{p}}{\epsilon_{p}}\right)
$$


for protons with energy $E_{p}$.

Based on the parameterisations we calculated the resulting summed electron and muon neutrino spectrum $\mathrm{N}_{\nu} / \mathrm{E}_{\nu}$ at the source $\left(\nu_{e}: \nu_{\mu}=1: 2\right)$. Assuming full mixing the muon neutrino spectrum at Earth is then given by one-third of $\mathrm{N}_{\nu} / \mathrm{E}_{\nu}$. The resulting muon neutrino spectrum is only a little dependent on the relative fraction of the electron and muon neutrinos at the source.

Considering spectra with $1.8<\alpha<3.0$ and $10 \mathrm{TeV}<\epsilon_{p}<1 \mathrm{PeV}$ and assuming full neutrino mixing, we find that the spectra of $\gamma$-rays and muon neutrinos at the Earth can be described by

$$
\frac{\mathrm{N}_{\gamma / \nu}}{\mathrm{E}_{\gamma / \nu}} \approx k_{\gamma / \nu}\left(\frac{E_{\gamma / \nu}}{1 \mathrm{TeV}}\right)^{-\Gamma_{\gamma / \nu}} \exp \left(-\sqrt{\frac{E_{\gamma / \nu}}{\epsilon_{\gamma / \nu}}}\right)
$$

and the parameters $k, \Gamma$, and $\epsilon$ are given by

$$
\begin{aligned}
k_{\nu} & \approx(0.71-0.16 \alpha) k_{\gamma} \\
\Gamma_{\nu} & \approx \Gamma_{\gamma} \approx \alpha-0.1 \\
\epsilon_{\nu} & \approx 0.59 \epsilon_{\gamma} \approx \epsilon_{p} / 40
\end{aligned}
$$

Figure 2 illustrates the accuracy of the parameterisations of the cut-off energy and the normalisation. Equation 4 provides a satisfactory fit to the $\gamma$-ray spectra of all sources detected using H.E.S.S., where for sources with no published claim of a curvature a pure power law is fitted $\left(\epsilon_{\gamma}=\infty\right)$. By refitting the spectral points (for references see Tab.11) to this equation we produce predicted muon neutrino spectra under the assumptions given above.

Figure 4 shows in the top plots as examples the neutrino spectra of two of the strongest Galactic $\gamma$-ray sources with observed cutoff, the SNR RX J1713.7-3946 and the PWN Vela X. The open and shaded areas of the $\gamma$-ray and neutrino spectra, respectively, include the $1 \sigma$ systematic uncertainties. This we assume to be $20 \%$ on the normalisation, $10 \%$ on the cutoff energy, and 0.1 on the index based on the systematic uncertainties of the published H.E.S.S. $\gamma$-ray spectra. Table 1 contains the parameters of the neutrino fluxes for all sources, where it should be noted that $\Gamma_{\nu}$ and $\epsilon_{\nu}$ are strongly correlated.

Some essential assumptions are made in the calculation of the predicted signal, and it is important to assess the validity of these assumptions on a source-by-source basis. The most important of these assumptions are

1. no significant contribution of non hadronic processes to the measured $\gamma$-ray signal; 
2. no significant $\gamma$-ray absorption within the source, i.e. radiation and matter densities are sufficiently low for most $\gamma \mathrm{s}$ to escape;

3. no significant $p \gamma$ interaction (radiation density low);

4. charged pions decay before interacting (matter density is low);

5. muons decay without significant energy loss (magnetic field is low);

6. nucleus-nucleus interactions produce pion spectra that are similar enough to the $p-p$ case that they can be treated in the same way;

7. the size of the emitting region within each source is large enough that oscillations will produce a fully mixed neutrino signal at the Earth $\left(\nu_{e}: \nu_{\mu}: \nu_{\tau}=1: 1: 1\right)$.

For all of the extended $\gamma$-ray sources detected by H.E.S.S., it seems likely that these conditions (with the likely exception of assumption 1 in several sources) are valid. One probable exception to assumptions 2 and 7 is the pointlike source LS5039, discussed in detail in Aharonian et al. (2006).

\section{Neutrino event rates}

\subsection{Signal event rates}

Given a neutrino spectrum $\stackrel{N}{\nu}_{\nu} / \mathrm{E}_{\nu}$ at the Earth from a source the event rate in a neutrino telescope can be calculated as

$$
\frac{\mathrm{N}_{\nu}}{\mathrm{t}}=\int \mathrm{E}_{\nu} A_{\nu}^{\mathrm{eff}} \frac{\mathrm{N}_{\nu}}{\mathrm{E}_{\nu}}
$$

Here, $A_{\nu}^{\text {eff }}$ is the neutrino effective area of the detector comprising the detection efficiency of neutrinos with an Earth-based telescope. Deep-sea neutrino telescopes detect neutrinos via the measurement of the Cherenkov light emitted by muons produced in the interaction

of high energy neutrinos. Muons produced in hadronic interactions of charged CR in the Earth's atmosphere present a background with a flux many orders of magnitude higher than the expected cosmic neutrino flux. To suppress this background neutrino telescopes are optimised to observe upward-going neutrinos, using the Earth as a filter. The neutrino attenuation in the Earth, as well as the neutrino conversion probability and the muon detection efficiency, are comprised in the effective area $A_{\nu}^{\mathrm{eff}}$. 
Due to the increase of the neutrino cross section and the muon range and its light yield per unit path length with energy the effective area is energy-dependent. Figure 3 shows the effective area of the ANTARES detector (Brunner et al. 2005) and the estimate for a future KM3NeT detector with an instrumented volume of $1 \mathrm{~km}^{3}$ (Kuch 2006) for muon neutrinos in the energy range from $10 \mathrm{GeV}$ to $1 \mathrm{PeV}$. (Since currently dedicated reconstruction software for the KM3NeT detector is not yet available, its effective area is calculated by requiring signals from the muon in at least 10 photo-sensors.) Absorption effects due to the increase of the neutrino cross section with energy and the resulting increasing opacity of the Earth start to affect the effective area at about $100 \mathrm{TeV}$ for small nadir angles between the detector normal and the source direction and are thus of no effect in the analysis described here (for the determination of the event numbers in Tab.1 the energy range was limited to $<100 \mathrm{TeV}$ ). Therefore, the effective area is assumed to be the same for all observation angles between the source and the detector. Both detectors operate at a neutrino reconstruction threshold of $\sim 100 \mathrm{GeV}$ well matched to the $\gamma$-ray threshold of H.E.S.S.

The detection of the muon-induced Cherenkov light with a three dimensional array of photo sensors allows one to reconstruct the flight direction and energy of the muon, which is used as an estimator for the flight direction and energy of the primary neutrino. In our study we neglect the effect of the uncertainty in the energy determination and use the true neutrino energy instead. The angular resolution $\sigma_{\mathrm{PSF}}$ of the neutrino direction for a deep-sea neutrino telescope decreases with increasing energy and is in the case of ANTARES better than $0.4^{\circ}$ for neutrino energies above $1 \mathrm{TeV}$. For KM3NeT a slightly better resolution can be expected, where in the energy range considered here the resolution is dominated by the difference in the flight direction of the neutrino and its muon. For the sake of simplicity we assume in our study angular resolutions of $0.4^{\circ}$ and $0.3^{\circ}$ for all energies for the ANTARES and KM3NeT detector, respectively. The optimal search window for an extended neutrino source, assuming an isotropic background of atmospheric neutrinos, is then given approximately as $\Theta_{\text {opt }}=1.6 \times \sqrt{\sigma_{\mathrm{PSF}}^{2}+\sigma_{\mathrm{src}}^{2}}$, where $\sigma_{\mathrm{src}}$ is the rms width of the source. This is also valid for the extended sources RX J1713.7-3946 and RX J0852.0-4622 which exhibit a ring-like region of enhanced emission. In these two cases convolutions of the point-spread function of the detector with the emission profiles yield distributions that can be approximated by Gaussian distributions with $\sigma=\sqrt{\sigma_{\mathrm{PSF}}^{2}+\sigma_{\mathrm{src}}^{2}}$. For the diffuse plane emission $\left(|b|<1^{\circ},|l|<30^{\circ}\right)$ we use a search window of size $60 \times 2 \mathrm{deg}^{2}$.

The number of observed events with a neutrino telescope is then calculated by

$$
N_{\nu}=R_{\Theta_{\mathrm{opt}}} \int \mathrm{t} \frac{\mathrm{N}_{\nu}}{\mathrm{t}}
$$

where the integration runs over the observation time when the source is below the horizon, and $R_{\Theta_{\mathrm{opt}}}=0.72$ is a reduction factor taking into account the loss of source neutrinos outside 
the search window (for the diffuse plane emission we use $R_{\Theta_{\mathrm{opt}}}=1$ ).

The differential fluxes of observed source neutrinos from RX J1713.7-3946 and Vela X

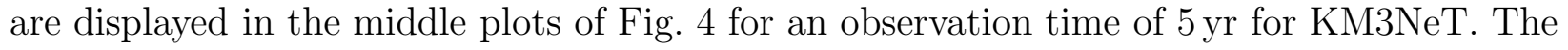
bottom two plots show the corresponding integrated numbers of events for neutrino energies $>E_{\nu}$. Table 1 shows the calculated neutrino event numbers of all Galactic $\gamma$-ray sources for $5 \mathrm{yr}$ of observation time with a $1 \mathrm{~km}^{3} \mathrm{KM} 3 \mathrm{NeT}$ detector for energies above 1 and $5 \mathrm{TeV}$. For each source, the range of possible neutrino event numbers and the mean neutrino event number (in parentheses) are displayed. The neutrino event number ranges result from the $1 \sigma$ error bands of the fits, taking into account the systematic uncertainty. For sources with no published claim of a curvature in the $\gamma$-ray spectrum, the lower bound of the neutrino event number results from the uncertainty of the fit of a curved spectrum, whereas the upper bound results from the uncertainty of the fit of a pure power law. We note that taking a pure power law instead of a curved spectrum might result in a considerable difference of expected neutrino rates. Fitting for example a pure power law for the SNR RX J1713.7-3946 results in 16.0 neutrinos in $5 \mathrm{yr}$ for KM3NeT compared to 10.7 for a curved spectrum (see also the two lower left plots in Fig.(4). For ANTARES, each of the brightest sources VelaX, RX J1713.7-3946, and RXJ0852.0-4622 yields at most 0.3 events in 5yr. Taking into account the different methods used, our calculations are in good agreement with recent

calculations of neutrino event rates from Galactic sources from Kistler \& Beacom (2006) and Distefano (2006).

\subsection{Background event rates}

Neutrinos produced in hadronic interactions of charged CRs in the atmosphere (atmospheric neutrinos) on the opposite side of the Earth result in signals in the telescope indistinguishable from those of cosmic neutrinos. The atmospheric neutrino event rate can be calculated in the same way as the source neutrino event rate. The flux of atmospheric neutrinos rapidly decreases with increasing energy and also strongly depends on the zenith angle, i.e. the time of the day. For large zenith angles the path length of the pions and muons in the thin outer atmosphere is larger compared to small zenith angles resulting in a higher decay probability and thus a larger neutrino flux. In this study we use the parameterisation of the atmospheric neutrino flux of Volkova (1980), which reasonably well parametrises the energy and zenith angle dependence in the energy range below $100 \mathrm{TeV}$. The measured size of the objects in $\mathrm{TeV} \gamma$-rays then allows us to give reliable estimates of the rates of detection of atmospheric neutrinos from the directions of $\gamma$-ray production regions.

The daily averaged atmospheric neutrino fluxes from sky regions of RX J1713.7-3946 
and Vela X integrated over the respective search windows are shown in Fig.4. Due to the steeper energy dependence of the atmospheric neutrino flux, the signal-to-background ratio improves with increasing energy. The calculated event numbers for atmospheric neutrinos for KM3NeT for $5 \mathrm{yr}$ are displayed in Tab.1, where we use a mean position $b=0^{\circ}, l=0^{\circ}$ in Galactic coordinates for the diffuse plane emission. For ANTARES these numbers are at least of the same size as the maximal numbers of detected source events.

\section{Discussion}

Sensitive TeV $\gamma$-ray detectors have surveyed most of our Galaxy, and it is likely that all of the bright Galactic $\gamma$-ray sources have now been identified. Most of these bright sources are located in the southern sky and are hence best visible (at energies below $\sim 100 \mathrm{TeV}$ ) to neutrino telescopes located in the Northern Hemisphere.

Under the assumption that the $\gamma$-rays are dominantly produced via $p-p$ interactions we use $\gamma$-ray spectra measured with H.E.S.S. to derive the associated neutrino emission. Our neutrino flux calculation differs from previous work in two important respects: the use of new parametrisations of the $\gamma$ and neutrino production in $p-p$ interactions and the use of curved $\gamma$-ray source spectra if such a claim is published.

We have calculated the expected neutrino event rates for two neutrino telescopes in the Mediterranean Sea: ANTARES (currently under construction) and KM3NeT, a future $\mathrm{km}^{3}$ scale detector. For the calculations we used neutrino telescope effective areas based on full simulations of the detector response and took into account the effect of optimal search windows.

We find that the brightest $\gamma$-ray sources produce neutrino rates above $1 \mathrm{TeV}$, comparable to the background from atmospheric neutrinos. The expected event rates of these sources in the ANTARES detector make a detection unlikely. For KM3NeT, with an instrumented volume of $1 \mathrm{~km}^{3}$, an event rate of a few neutrinos per year $\left(E_{\nu}>1 \mathrm{TeV}\right)$ from each of the three brightest $\gamma$-ray sources (RX J1713.7-3946, VelaX, and RX J0852-4622) can be expected, and the detection of individual sources seems possible after several years of stable detector operation (see Table 1). However, because of the low statistics of source neutrinos the detection of $\mathrm{TeV}$ signals from a major fraction of H.E.S.S. sources will be difficult for $1 \mathrm{~km}^{3}$ class neutrino telescopes.

We would like to point out that the event rates presented in this paper are based on preliminary calculations of the KM3NeT effective neutrino area, which, e.g., do not include the muon reconstruction procedure (see Sec.4.1). Therefore, future more detailed 
calculations will probably lead to a reduction of the effective area. Also, we want to note that the estimated rates should be treated as upper limits for most sources, since a significant contribution from directly accelerated electrons to the $\gamma$-ray fluxes is possible (or likely). On the other hand, the neutrino rates from sources in which $\gamma$-rays are heavily absorbed could be significantly (up to orders of magnitude) higher than those given here. X-ray binaries represent one source class in which such absorption is likely.

When this paper was completed we learned that Vissani (2006) published a calculation of neutrino event rates for RX J1713.7-3946 in which he also uses a primary spectrum with a cut-off at high energies. The quoted event numbers are in good agreement with those given in this paper taking into account the differences in the two analyses.

J. H. acknowledges the support of the German BMBF through Verbundforschung AstroTeilchenphysik (05CH5VH1/0).

\section{REFERENCES}

Aharonian, F. A., 2001, Space Sci. Rev., 99, 187

Aharonian, F. A. \& Neronov, A., 2005, Ap\&SS, 300, 255

Aharonian, F. A., Anchordoqui, L. A., Khangulyan, D. \& Montaruli, T., 2006, J. Phys. Conf. Series, 39,408

Alvarez-Muñiz, J. \& Halzen, F., 2002, ApJ, 576, L33

AMANDA Coll., Ackermann, M. et al., 2005, Phys. Rev., D71, 077102

Amato, E., Guetta, D. \& Blasi, P., 2003, A\&A, 402, 827

ANTARES Coll., Aslanides, E. et al., preprint (astro-ph/9907432)

BAIKAL Coll., Balkanov, V. A et al., 1999, Astropart. Phys., 12, 75

Bednarek, W., 2003, A\&A, 407, 1

Berezinsky, V.S. \& Volynsky, V. V., 1979, Proc. 16th ICRC, Kyoto, 10, 332

Brogan, C. L. et al., 2005, ApJ, 629, L105

Brunner, J. et al. (ANTARES Coll.), 2005, Nucl. Phys., B145, 323

Costantini, M. L., Vissani, F., 2005, Astropart. Phys., 23, 477 
Crocker, R. M., Melia, F. \& Volkas, R.R., 2005a, ApJ, 622, L37

Crocker, R. M. et al. 2005b, ApJ, 622, 892

Dame, T. M., Hartmann, D. \& Thaddeus, P., 2001, ApJ, 547, 792

Dickinson, J. E. et al., 2000, Nucl. Instrum. Method., A440, 95

Distefano, C., 2006, in Proceedings of the Workshop on Multi-Messenger Approach to Unidentified Gamma-Ray Sources, (Springer: Ap\&SS Special Suppl.), in press, preprint (astro-ph/0608514)

Fletcher, R.S., Gaisser, T. K., Lipari, P. \& Stanev, T., 1994, Phys. Rev., D50, 5710

Gaisser, T. K., 1990, 'Cosmic Rays and Particle Physics', Cambridge University Press

HEGRA Coll., Aharonian, F. A. et al., 2001, A\&A, 370, 112

HEGRA Coll., Aharonian, F. A. et al., 2004, ApJ, 614, 897

HEGRA Coll., Aharonian, F. A. et al., 2005, A\&A, 431, 197

H.E.S.S. Coll., Aharonian, F. A. et al., 2004a, A\&A, 425, L13

H.E.S.S. Coll., Aharonian, F. A. et al., 2004b, Nature, 432, 75

H.E.S.S. Coll., Aharonian, F. A. et al., 2005a, A\&A, 432, L25

H.E.S.S. Coll., Aharonian, F. A. et al., 2005b, Science, 307, 1938

H.E.S.S. Coll., Aharonian, F. A. et al., 2005c, Science, 309, 746

H.E.S.S. Coll., Aharonian, F. A. et al., 2005d, A\&A, 435, L17

H.E.S.S. Coll., Aharonian, F. A. et al., 2005e, A\&A, 437, L7

H.E.S.S. Coll., Aharonian, F. A. et al., 2005f, A\&A, 437, 135

H.E.S.S. Coll., Aharonian, F. A. et al., 2005g, A\&A, 439, 1013

H.E.S.S. Coll., Aharonian, F. A. et al., 2005h, A\&A, 442, 1

H.E.S.S. Coll., Aharonian, F. A. et al., 2006a, ApJ, 636, 777

H.E.S.S. Coll., Aharonian, F. A. et al., 2006b, Nature, 439, 695

H.E.S.S. Coll., Aharonian, F. A. et al., 2006c, A\&A, 448, L43 
H.E.S.S. Coll., Aharonian, F. A. et al., 2006d, A\&A, 449, 223

H.E.S.S. Coll., Aharonian, F. A. et al., 2006e, A\&A, 456, 245

H.E.S.S. Coll., Aharonian, F. A. et al., 2006f, preprint (astro-ph/0607192)

H.E.S.S. Coll., Aharonian, F. A. et al., 2006g, A\&A, 457, 899

H.E.S.S. Coll., Aharonian, F. A. et al., 2006h, A\&A, in press, preprint (astro-ph/0607548)

H.E.S.S. Coll., Aharonian, F. A. et al., 2007, A\&A, in press, preprint (astro-ph/0612495)

Hillas, A. M., 2005, J. Phys., G31, R95

Hinton, J. A. 2004, NewA Rev., 48, 331

Horns, D. et al, 2006, A\&A, 451, L51

Hoshino, M. et al., 1992, ApJ, 390, 454

Hunter et al., 1997, ApJ, 481, 205

Katz, U. F., 2006, Nucl. Instrum. Method., A567, 457

Kelner, S. R., Aharonian, F. A., Bugayov, V. V., 2006, Phys. Rev., D74, 034018

Kistler, M. D. \& Beacom, J.F., 2006, Phys. Rev. D, 74, 063007

Kuch, S., 2006, 2006, Nucl. Instrum. Method., A567, 498

Lipari, P., 2005, 2006, Nucl. Instrum. Method., A567, 405

MAGIC Coll., Albert, J. et al., 2006, Science, 312, 1771

McKay, T.A. et al., 1993, ApJ, 417, 742

MILAGRO Coll., Atkins, R. et al., 2005, Phys. Rev. Lett., 95, 251103

Saz Parkinson, P. M. et al. (MILAGRO Coll.), 2004, eConf C041213:2418, preprint (astro-ph/0503244)

van Stekelenborg, J. et al., 1993, Phys. Rev., D48, 4504

Strong, A. W., Moskalenko, I. V. \& Reimer, O., 2004, ApJ, 613, 962

Ubertini, P. et al., 2005, ApJ, 629, L109

Vissani, F., 2006, Astropart. Phys., 26, 310 
Volkova, L. V., 1980, Soviet. J. Nucl. Phys., 31(6), 784 


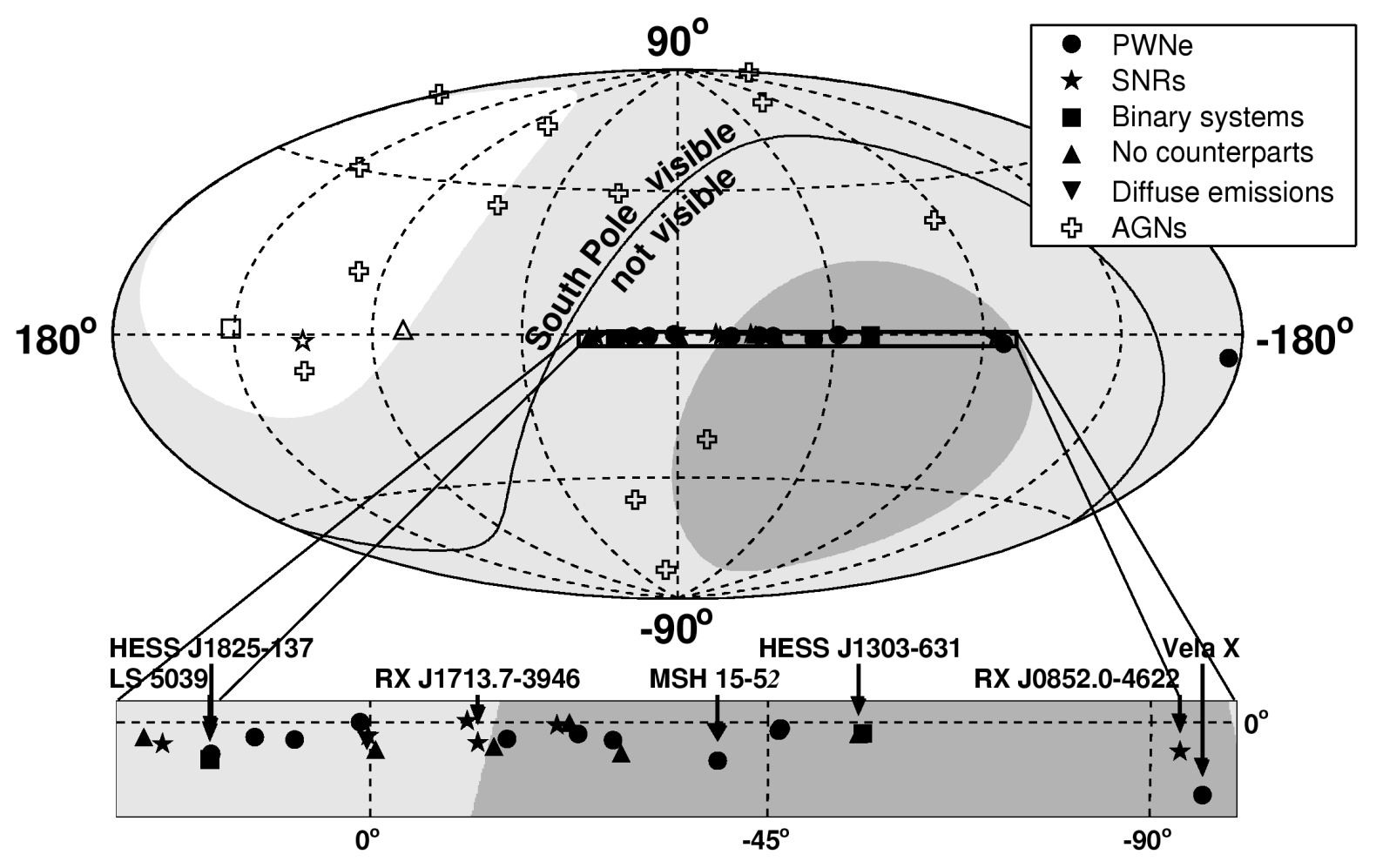

Fig. 1.- Sky map of $\mathrm{TeV} \gamma$-ray sources in Galactic coordinates together with their visibility to neutrino telescopes. Source types are represented by different symbols: filled symbols mark sources discussed in this paper, the shaded regions represent $25 \%-75 \%$ (light gray) and $>75 \%$ (dark gray) visibility to a detector in the central Mediterranean Sea for energies below $\sim 100 \mathrm{TeV}$, and the solid curve shows the extent of the visibility of a South Pole detector (IceCube). The inset displays a zoomed view of the area between $-100^{\circ}$ and $+28^{\circ}$ in Galactic longitude and about $-3.5^{\circ}$ and $+0.8^{\circ}$ in Galactic latitude. 


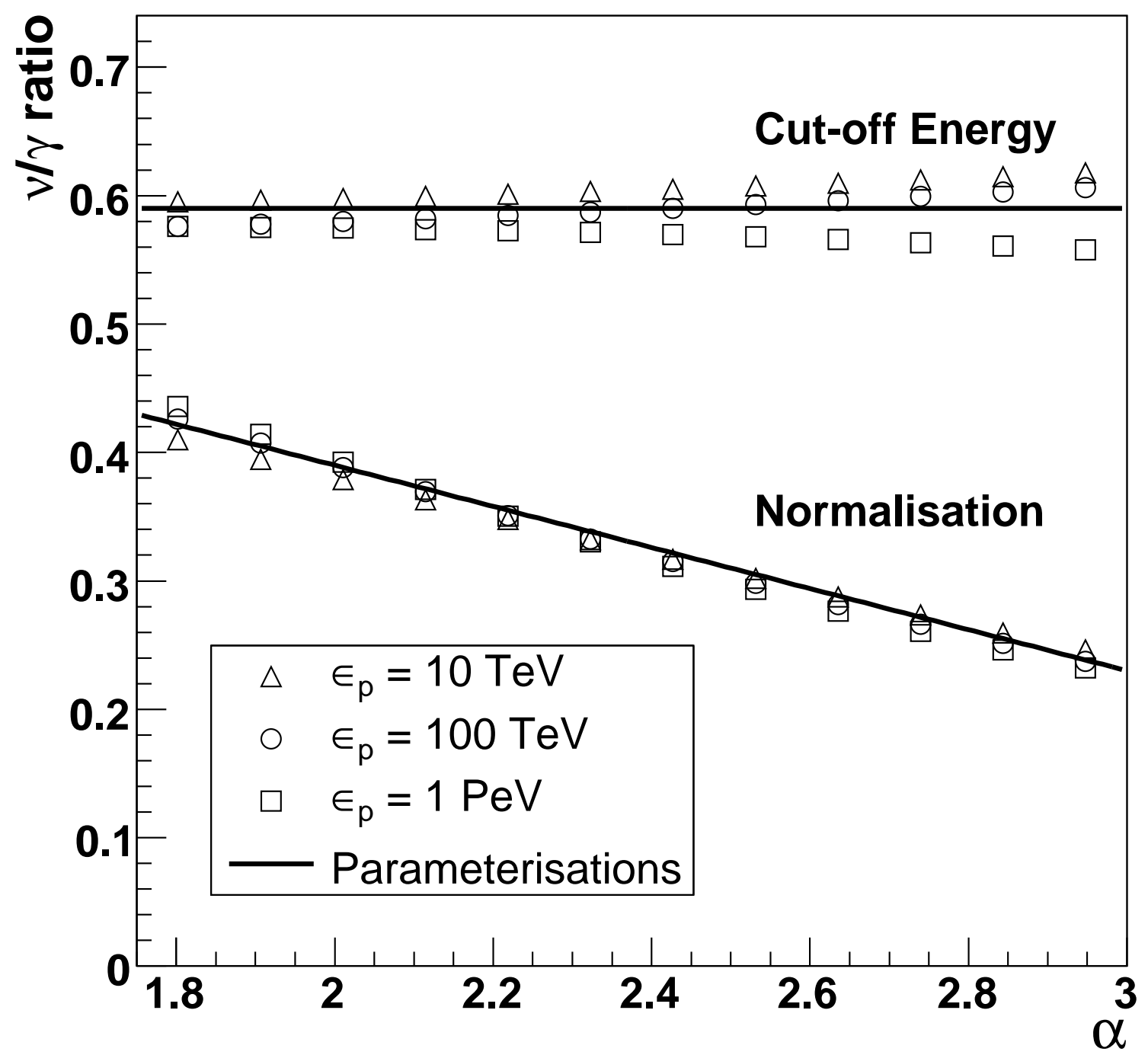

Fig. 2.- Ratio of the best-fit neutrino and $\gamma$-ray normalisations $\left(k_{\nu} / k_{\gamma}\right)$ and cut-off energies $\left(\epsilon_{\nu} / \epsilon_{\gamma}\right)$ for a range of proton indices $\alpha$ and cutoff energies $\epsilon_{p}$. The indices of the secondary spectra are kept fixed at $\alpha-0.1$. The curves show the parameterisations given in the text. 


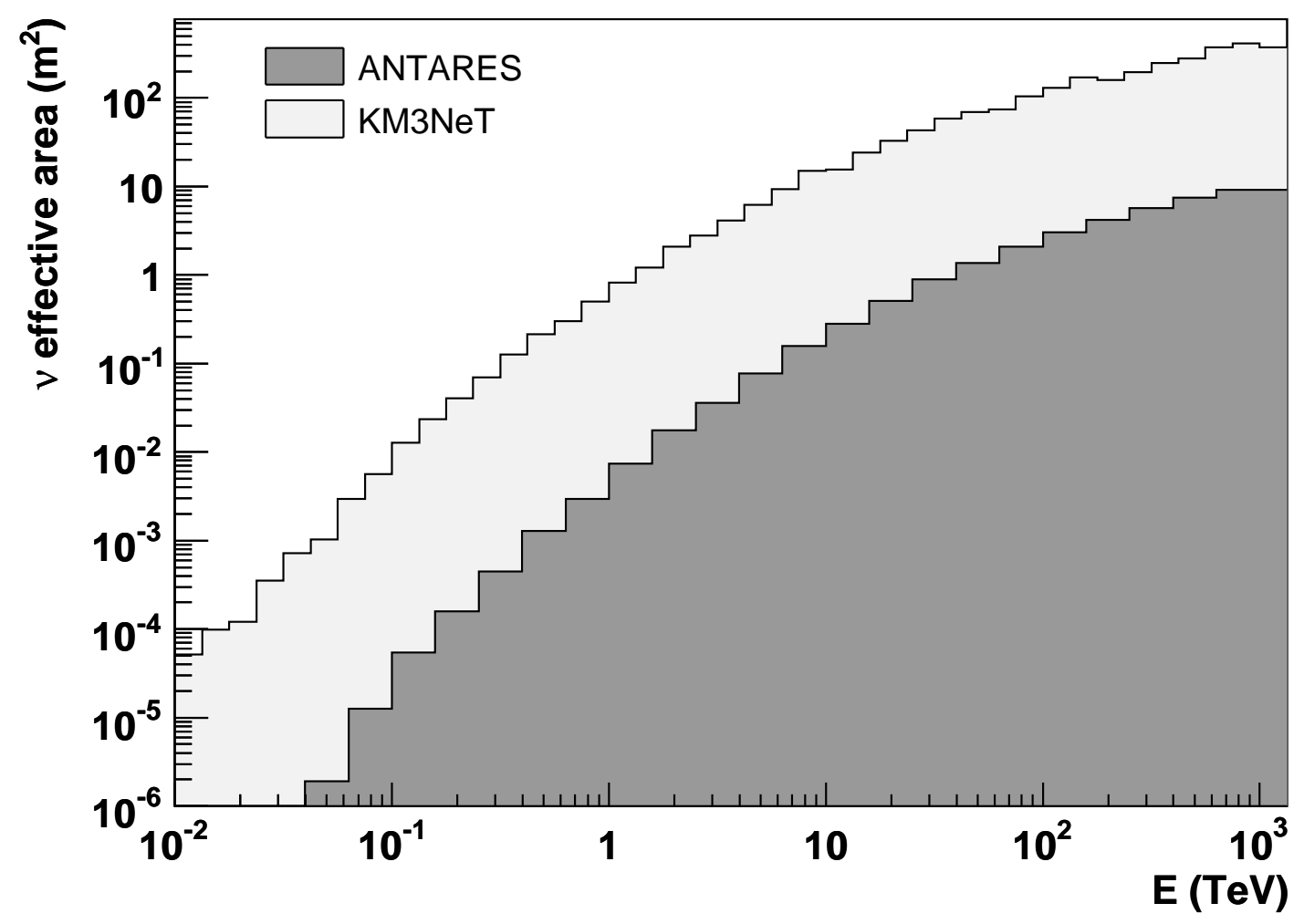

Fig. 3.- Effective areas $A_{\nu}^{\mathrm{eff}}\left(E_{\nu}\right)$ for muon neutrinos of the ANTARES detector and the KM3NeT detector with an instrumented volume of $1 \mathrm{~km}^{3}$ in the energy range from $10 \mathrm{GeV}$ to $1 \mathrm{PeV}$. 

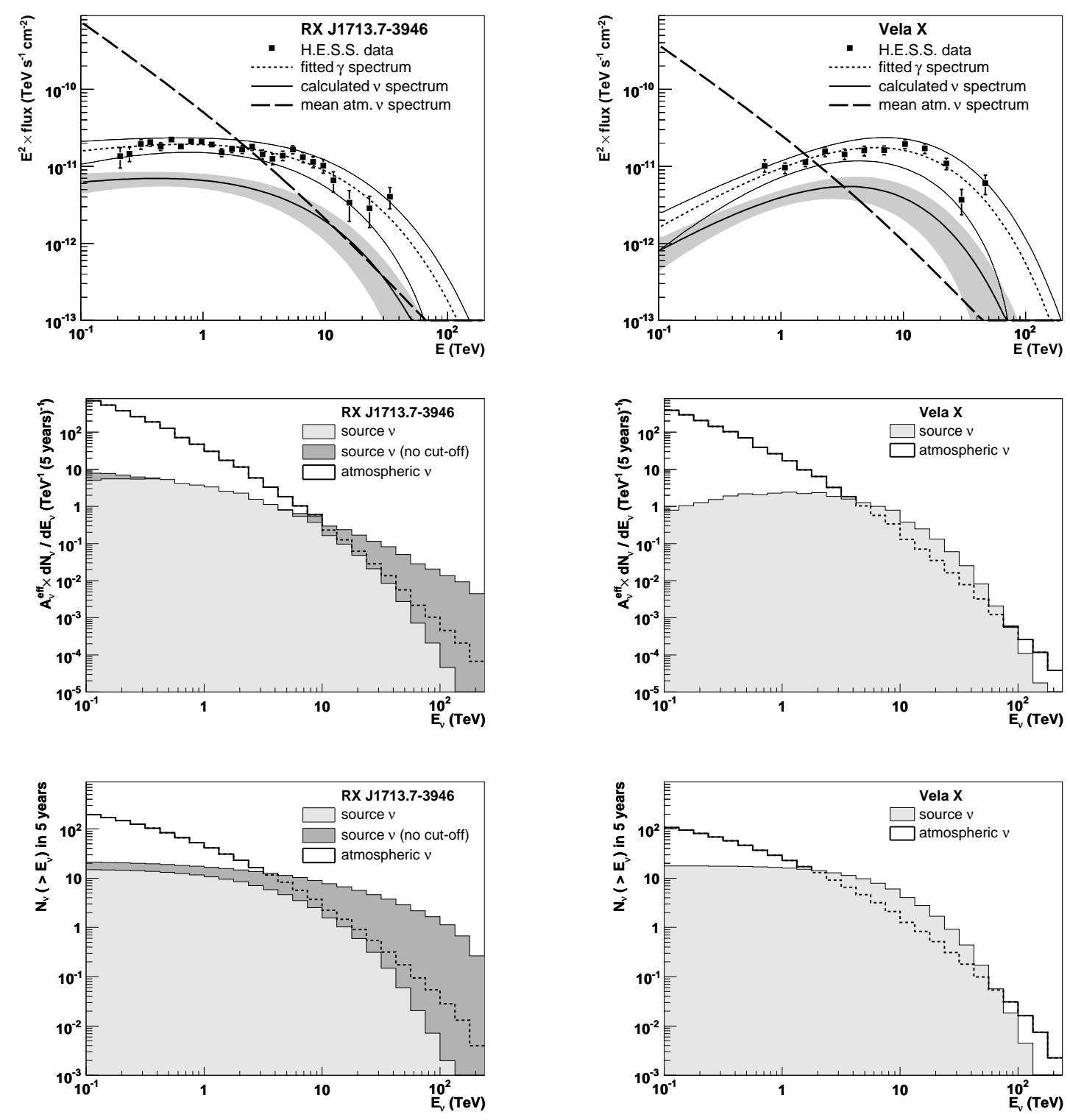

Fig. 4.- Left: Plots for RX J1713.7-3946, Right: Plots for Vela X. Top: Measured $\gamma$-ray flux and the estimated neutrino flux with their error bands, together with the atmospheric neutrino flux. The daily averaged atmospheric neutrino flux is integrated over the search window. Middle: Differential flux of source neutrinos in $5 \mathrm{yr}$, together with the corresponding atmospheric neutrino flux for KM3NeT. Bottom: Number of detected events with neutrino energies $>E_{\nu}$ in $5 \mathrm{yr}$ in the KM3NeT detector. In addition, for RX J1713.7-3946 the lower two plots show the case for a source spectrum assuming no cut-off. In the lower four plots the limited size of the search window is taken into account, reducing the number of source neutrinos compared to the upper two plots. 
Table 1: Shown are H.E.S.S. catalog sources with the range of estimated neutrino event rates and mean rates (in parentheses) within the search window in KM3NeT for 5 years of operation together with the expected atmospheric neutrino background for neutrino energies above $1 \mathrm{TeV}$ and $5 \mathrm{TeV}$ (for definition of range and search window see Sec.41). The reference for each source is listed in column Ref. (all H.E.S.S. Coll.). The values under $\varnothing$ represent the diameter of the $\gamma$-ray source. In the case of non circular sources, the corresponding dimensions in degrees are given. The column Vis. displays the visibility (fraction of time when the source is below the horizon) of the source to KM3NeT, where for the diffuse plane emission a mean visibility of $65 \%$ is assumed. The neutrino spectrum parameters at the Earth, $k_{\nu}$ and $\epsilon_{\nu}$, are given in units of $10^{-12} \mathrm{TeV}^{-1} \mathrm{~cm}^{-2} \mathrm{~s}^{-1}$ and $\mathrm{TeV}$, respectively. A missing entry for $\epsilon_{\nu}$ indicates that a pure power law was fitted to the $\gamma$-ray data points of the respective source. Also, note that $\Gamma_{\nu}$ and $\epsilon_{\nu}$ are strongly correlated.

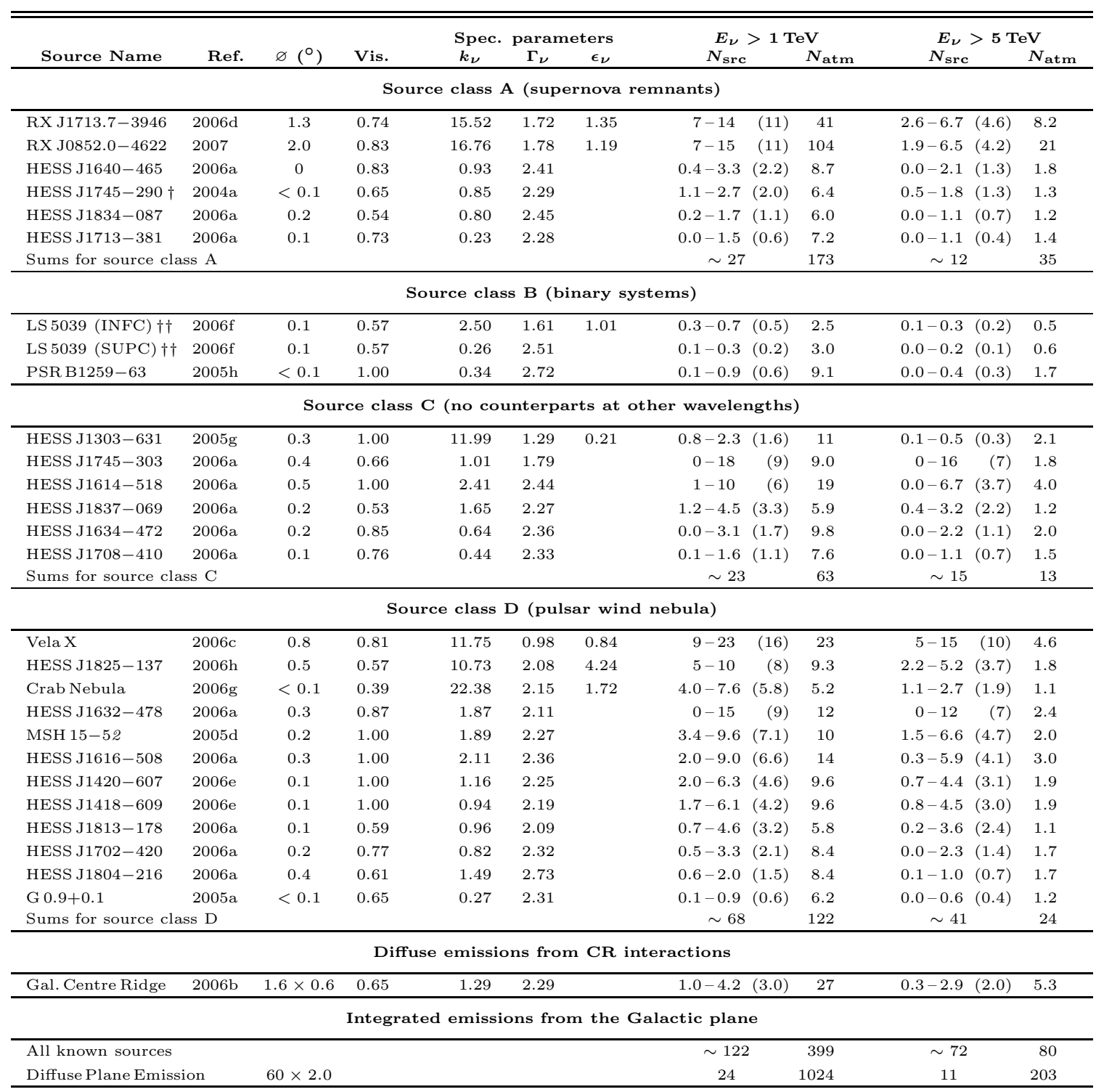

